\title{
Iterative methods for ternary diffusions
}

\author{
Henryk Leszczyński* and Monika Wrzosek
}

${ }^{\text {*Correspondence: }}$

hleszcz@mat.ug.edu.pl

Institute of Mathematics, University

of Gdańsk, Wita Stwosza 57, Gdańsk,

80-952, Poland

\begin{abstract}
We apply iterative methods to three-component diffusion equations and study their convergence in $L^{2}$ and in the Sobolev space $W^{1, \infty}$. The system is parabolic and mass-conservative. Newton's method converges very fast and its iterations do not leave the set of admissible functions.
\end{abstract}

MSC: $35 \mathrm{~K} 51 ; 35 \mathrm{~K} 57 ; 65 \mathrm{M} 12 ; 65 \mathrm{M} 80$

Keywords: diffusion; iterative method; Newton's method

\section{Introduction}

Since its discovery and later analysis by Darken [1], the Kirkendall effect [2] has been found in various alloy systems, and studies on lattice defects and diffusion developed significantly. The Danielewski-Holly method [3] extends the Darken standard theory of interdiffusion and describes the process in the bounded mixture showing constant concentration. Under certain regularity assumptions and quantitative condition Danielewski and Holly proved the existence and uniqueness of solution to PDE describing the interdiffusion phenomena. Further developments have been presented in numerous articles; e.g. [4,5].

In the paper we apply Newton's method (see [6-8]) to three-component diffusion equations and study the convergence in $L^{2}$ and Sobolev space $W^{1, \infty}$. The system of equations is strongly coupled, however, the maximum principle presented in Section 1 confirms its parabolic type. Parabolicity is additionally confirmed by our convergence result for iterative methods. This falsifies the nonparabolicity hypothesis by Danielewski and Holly [3], where they construct an initial concentration whose $L^{2}$ norm increases in time, at least on some interval. The Newton method, known as quasilinearization method, is very useful in modern numerical methods for solving PDE's; see [9]. We apply this method to strongly coupled parabolic systems describing diffusing mixtures. This strong parabolicity might have caused weird phenomena, but we have discovered a kind of maximum principle and some conservation laws in this system, hence the iterative methods proposed here behave very well. Our result is very useful in numerical simulations when one wants to construct reliable and fast convergent approximations. Since Newton's method produces linear PDE's satisfying maximum principles and a priori estimates of the respective Green functions or Cauchy kernels, one can find errors estimates much better than those obtained from the Newton-Kantorovich theorem, cf. [10,11].

Consider a mixture composed of three different components. Let $t \geq 0, x \in[-L, L]$, $v_{i}:[0, \infty) \times[-L, L] \rightarrow \mathbb{R}$ denote the velocity field of the $i$ th component and $c_{i}:[0, \infty) \times$ $[-L, L] \rightarrow \mathbb{R}$ its molar density or molar concentration. It is a measure of the number of 
particles contained in any volume, $c_{1}+c_{2}+c_{3} \equiv$ const. The component diffusion flux is a Fickian flow:

$$
J_{i}^{d}(t, x):=-D_{i} \operatorname{grad} c_{i}
$$

where $D_{i}$ is the intrinsic diffusitivity of the $i$ th component which we assume to satisfy $D_{1}>D_{2}>D_{3}>0$. Denote $D_{i}^{\prime}:=D_{i}-D_{3}$ for $i=1,2$. The overall $i$ th component flux is a sum of diffusion and convection fluxes:

$$
J_{i}:=J_{i}^{d}+c_{i} v^{D}
$$

where $v^{D}$ stands for a drift velocity. By the mass conservation law:

$$
\frac{\partial c_{i}}{\partial t}=-\operatorname{div} J_{i}
$$

and upon denoting $u=c_{1}, v=c_{2}, w=c_{3}$ we arrive at the following system of equations:

$$
\begin{aligned}
& u_{t}=D_{1} u_{x x}-\left(u\left[D_{1}^{\prime} u_{x}+D_{2}^{\prime} v_{x}\right]\right)_{x}, \\
& v_{t}=D_{2} v_{x x}-\left(v\left[D_{1}^{\prime} u_{x}+D_{2}^{\prime} v_{x}\right]\right)_{x} \\
& w_{t}=D_{3} w_{x x}-\left(w\left[D_{1}^{\prime} u_{x}+D_{2}^{\prime} v_{x}\right]\right)_{x}
\end{aligned}
$$

with the initial condition

$$
u(0, x)=u_{0}(x), \quad v(0, x)=v_{0}(x), \quad w(0, x)=w_{0}(x)=1-u_{0}(x)-v_{0}(x)
$$

for $x \in[-L, L]$ and the Neumann boundary condition

$$
\frac{\partial u}{\partial n}=0, \quad \frac{\partial v}{\partial n}=0, \quad \frac{\partial w}{\partial n}=0 \quad \text { for } t \geq 0, x \in\{-L, L\} .
$$

Let $\mathcal{X}$ denote the space consisting of triples of functions $(u, v, w)$ satisfying

$$
u, v, w \in C^{1,2} \text {, }
$$$$
u_{x}, u_{x x}, v_{x}, v_{x x} \text { are bounded, }
$$$$
u \geq 0, v \geq 0, w \geq 0 \text { for } t \geq 0, x \in[-L, L],
$$$$
u+v+w=1 \text { for } t \geq 0, x \in[-L, L]
$$

$u, v, w$ obey the Neumann boundary condition.

Remark 1.1 If $(u, v, w) \in \mathcal{X}$ then the third equation of (1.1) is not necessary, since $w=1-$ $u-v$. However, we keep it for a more convenient analysis of some properties of solutions.

Remark 1.2 We call

$$
v^{D}=D_{1}^{\prime} u_{x}+D_{2}^{\prime} v_{x}=D_{1} u_{x}+D_{2} v_{x}+D_{3} w_{x}
$$

the drift velocity; it describes the marker position. 
Lemma 1.3 (Mass conservation) If $(u, v, w) \in \mathcal{X}$ satisfy (1.1), (1.2) then

$$
\int_{-L}^{L} u d x=\text { const. }, \quad \int_{-L}^{L} v d x=\text { const. }, \quad \int_{-L}^{L} w d x=\text { const. }
$$

Proof The relation

$$
\frac{d}{d t} \int_{-L}^{L} u d x=0
$$

can be shown by means of the Neumann boundary condition.

Lemma 1.4 (Maximum principle) Suppose that $u(0, \cdot), v(0, \cdot), w(0, \cdot) \in C^{2}$ and

$$
u(0, x) \geq 0, \quad v(0, x) \geq 0, \quad w(0, x) \geq 0, \quad u(0, x)+v(0, x)+w(0, x)=1
$$

for $x \in[-L, L]$. If $(u, v, w)$ satisfy (1.1)-(1.3) then $(u, v, w) \in \mathcal{X}$.

Proof Let $\tilde{u}=u+\varepsilon e^{\lambda t}, \tilde{v}=v+\varepsilon e^{\lambda t}, \tilde{w}=w+\varepsilon e^{\lambda t}$ for $\varepsilon>0$. We have

$$
\begin{array}{llr}
\tilde{u}_{t}=u_{t}+\varepsilon \lambda e^{\lambda t}, & \tilde{u}_{x}=u_{x}, & \tilde{u}_{x x}=u_{x x}, \\
\tilde{v}_{t}=v_{t}+\varepsilon \lambda e^{\lambda t}, & \tilde{v}_{x}=v_{x}, & \tilde{v}_{x x}=v_{x x} .
\end{array}
$$

There exists $\lambda \in \mathbb{R}$ (sufficiently large) such that we have strong differential inequalities:

$$
\begin{aligned}
& \tilde{u}_{t}>D_{1} \tilde{u}_{x x}-\tilde{u}\left[D_{1}^{\prime} \tilde{u}_{x x}+D_{2}^{\prime} \tilde{v}_{x x}\right]-\tilde{u}_{x}\left[D_{1}^{\prime} \tilde{u}_{x}+D_{2}^{\prime} \tilde{v}_{x}\right], \\
& \tilde{v}_{t}>D_{2} \tilde{v}_{x x}-\tilde{v}\left[D_{1}^{\prime} \tilde{u}_{x x}+D_{2}^{\prime} \tilde{v}_{x x}\right]-\tilde{v}_{x}\left[D_{1}^{\prime} \tilde{u}_{x}+D_{2}^{\prime} \tilde{v}_{x}\right], \\
& \tilde{w}_{t}>D_{3} \tilde{w}_{x x}-\tilde{w}\left[D_{1}^{\prime} \tilde{u}_{x x}+D_{2}^{\prime} \tilde{v}_{x x}\right]-\tilde{w}_{x}\left[D_{1}^{\prime} \tilde{u}_{x}+D_{2}^{\prime} \tilde{v}_{x}\right] .
\end{aligned}
$$

We claim that $\tilde{u}>0, \tilde{v}>0, \tilde{w}>0$ in the whole domain. Suppose that this is not true and take the smallest $t^{*}>0$ such that $\tilde{u}\left(t^{*}, x^{*}\right)=0$, or $\tilde{v}\left(t^{*}, x^{*}\right)=0$, or $\tilde{w}\left(t^{*}, x^{*}\right)=0$ for some $x^{*} \in[-L, L]$. Without loss of generality we assume $\tilde{u}\left(t^{*}, x^{*}\right)=0$. Since $\tilde{u}\left(t^{*}, x^{*}\right)=$ $\min _{\left\{t \leq t^{*}, x \in[-L, L]\right\}} \tilde{u}(t, x)$ we have $\tilde{u}_{x}\left(t^{*}, x^{*}\right)=0, \tilde{u}_{t}\left(t^{*}, x^{*}\right) \leq 0$ and $\tilde{u}_{x x}\left(t^{*}, x^{*}\right) \geq 0$. Hence

$$
\begin{aligned}
0 \geq & \tilde{u}_{t}\left(t^{*}, x^{*}\right)>D_{1} \tilde{u}_{x x}\left(t^{*}, x^{*}\right)-\tilde{u}\left(t^{*}, x^{*}\right)\left[D_{1}^{\prime} \tilde{u}_{x x}\left(t^{*}, x^{*}\right)+D_{2}^{\prime} \tilde{v}_{x x}\left(t^{*}, x^{*}\right)\right] \\
& -\tilde{u}_{x}\left(t^{*}, x^{*}\right)\left[D_{1}^{\prime} \tilde{u}_{x}\left(t^{*}, x^{*}\right)+D_{2}^{\prime} \tilde{v}_{x}\left(t^{*}, x^{*}\right)\right] \geq 0,
\end{aligned}
$$

which is a contradiction. Thus $\tilde{u}>0$ for $t \geq 0, x \in[-L, L]$. If $\varepsilon \rightarrow 0^{+}$then $\tilde{u} \rightarrow u$. Hence $u>0$. Similarly, $v(t, x) \geq 0$ and $w(t, x) \geq 0$ for $t \geq 0, x \in[-L, L]$.

\section{Uniqueness}

Let $\overline{\mathcal{X}}$ be the closure of $\mathcal{X}$ w.r.t. the $L^{2}$ norm. The existence and uniqueness of solutions to problem (1.1)-(1.3) in $\mathcal{X}$ w.r.t. the Sobolev norm $W^{1,2}$ is given in [3]. The following proposition concerns the uniqueness of solutions in $L^{2}$. Since the set of $C^{2}$-functions is dense in $L^{2}$, the proof is carried out in $\mathcal{X}$. The uniqueness is obtained for weak solutions. 
Proposition 2.1 Assume that $(7-4 \sqrt{3}) D_{2} \leq D_{1} \leq(7+4 \sqrt{3}) D_{2}$ and $\left(u_{0}, v_{0}, w_{0}\right) \in \overline{\mathcal{X}}$. Then a weak solution $(u, v, w) \in \overline{\mathcal{X}}$ to problem (1.1)-(1.3) is unique in $L^{2}$.

Proof Since every $L^{2}$-function can be approximated by a sequence of $\mathcal{X}$-functions, it suffices to show the uniqueness of $\mathcal{X}$-solutions w.r.t. the $L^{2}$-norm. Let $(u, v, w) \in \mathcal{X}$ and $(\bar{u}, \bar{v}, \bar{w}) \in \mathcal{X}$ be solutions to (1.1)-(1.3). Denote

$$
\Delta u=u-\bar{u}, \quad \Delta v=v-\bar{v}, \quad \Delta w=w-\bar{w}
$$

and observe that

$$
\begin{aligned}
& \Delta u_{t}=D_{1} \Delta u_{x x}-\left(\Delta u\left[D_{1}^{\prime} u_{x}+D_{2}^{\prime} v_{x}\right]\right)_{x}-\left(\bar{u}\left[D_{1}^{\prime} \Delta u_{x}+D_{2}^{\prime} \Delta v_{x}\right]\right)_{x} \\
& \Delta v_{t}=D_{2} \Delta v_{x x}-\left(\Delta v\left[D_{1}^{\prime} u_{x}+D_{2}^{\prime} v_{x}\right]\right)_{x}-\left(\bar{v}\left[D_{1}^{\prime} \Delta u_{x}+D_{2}^{\prime} \Delta v_{x}\right]\right)_{x} \\
& \Delta w_{t}=D_{3} \Delta w_{x x}-\left(\Delta w\left[D_{1}^{\prime} u_{x}+D_{2}^{\prime} v_{x}\right]\right)_{x}-\left(\bar{w}\left[D_{1}^{\prime} \Delta u_{x}+D_{2}^{\prime} \Delta v_{x}\right]\right)_{x} .
\end{aligned}
$$

We have

$$
\begin{aligned}
\int_{-L}^{L} \Delta u \Delta u_{t} d x= & D_{1} \int_{-L}^{L} \Delta u \Delta u_{x x} d x-\int_{-L}^{L} \Delta u\left(\Delta u\left[D_{1}^{\prime} u_{x}+D_{2}^{\prime} v_{x}\right]\right)_{x} d x \\
& -\int_{-L}^{L} \Delta u\left(\bar{u}\left[D_{1}^{\prime} \Delta u_{x}+D_{2}^{\prime} \Delta v_{x}\right]\right)_{x} d x
\end{aligned}
$$

Using integration by parts we obtain

$$
\begin{aligned}
& 2 D_{1} \int_{-L}^{L} \Delta u \Delta u_{x x} d x=-2 D_{1} \int_{-L}^{L}\left(\Delta u_{x}\right)^{2} d x \\
& 2 \int_{-L}^{L} \Delta u\left(\Delta u\left[D_{1}^{\prime} u_{x}+D_{2}^{\prime} v_{x}\right]\right)_{x} d x=\int_{-L}^{L}(\Delta u)^{2}\left[D_{1}^{\prime} u_{x x}+D_{2}^{\prime} v_{x x}\right] d x \\
& 2 \int_{-L}^{L} \Delta u\left(\bar{u}\left[D_{1}^{\prime} \Delta u_{x}+D_{2}^{\prime} \Delta v_{x}\right]\right)_{x} d x=-2 \int_{-L}^{L} \Delta u_{x} \bar{u}\left[D_{1}^{\prime} \Delta u_{x}+D_{2}^{\prime} \Delta v_{x}\right] d x
\end{aligned}
$$

Hence

$$
\begin{aligned}
\frac{d}{d t} \int_{-L}^{L}\left[(\Delta u)^{2}+(\Delta v)^{2}+(\Delta w)^{2}\right] d x \\
=2 \int_{-L}^{L}\left(\Delta u \Delta u_{t}+\Delta v \Delta v_{t}+\Delta w \Delta w_{t}\right) d x \\
=-2 \int_{-L}^{L}\left(D_{1}\left(\Delta u_{x}\right)^{2}+D_{2}\left(\Delta v_{x}\right)^{2}+D_{3}\left(\Delta w_{x}\right)^{2}\right) d x \\
\quad-\int_{-L}^{L}\left((\Delta u)^{2}+(\Delta v)^{2}+(\Delta w)^{2}\right)\left[D_{1}^{\prime} u_{x x}+D_{2}^{\prime} v_{x x}\right] d x \\
\quad+2 \int_{-L}^{L}\left(\Delta u_{x} \bar{u}+\Delta v_{x} \bar{v}+\Delta w_{x} \bar{w}\right)\left[D_{1}^{\prime} \Delta u_{x}+D_{2}^{\prime} \Delta v_{x}\right] d x .
\end{aligned}
$$


By the fact that $\Delta w_{x}=-\Delta u_{x}-\Delta v_{x}$ we obtain

$$
\begin{aligned}
2 \int_{-L}^{L}\left(\Delta u \Delta u_{t}+\Delta v \Delta v_{t}+\Delta w \Delta w_{t}\right) d x \\
=-\int_{-L}^{L}\left((\Delta u)^{2}+(\Delta v)^{2}+(\Delta w)^{2}\right)\left[D_{1}^{\prime} u_{x x}+D_{2}^{\prime} v_{x x}\right] d x \\
\quad-2 \int_{-L}^{L}\left(D_{1}+D_{3}-D_{1}^{\prime}(\bar{u}-\bar{w})\right)\left(\Delta u_{x}\right)^{2} d x \\
\quad-2 \int_{-L}^{L}\left(D_{2}+D_{3}-D_{2}^{\prime}(\bar{v}-\bar{w})\right)\left(\Delta v_{x}\right)^{2} d x \\
\quad-2 \int_{-L}^{L}\left(2 D_{3}-D_{1}^{\prime}(\bar{v}-\bar{w})-D_{2}^{\prime}(\bar{u}-\bar{w})\right) \Delta u_{x} \Delta v_{x} d x
\end{aligned}
$$

We examine the nonnegative definiteness of the matrix:

$$
A=\left[\begin{array}{cc}
D_{1}+D_{3}-D_{1}^{\prime}(\bar{u}-\bar{w}) & D_{3}-\frac{1}{2} D_{1}^{\prime}(\bar{v}-\bar{w})-\frac{1}{2} D_{2}^{\prime}(\bar{u}-\bar{w}) \\
D_{3}-\frac{1}{2} D_{1}^{\prime}(\bar{v}-\bar{w})-\frac{1}{2} D_{2}^{\prime}(\bar{u}-\bar{w}) & D_{2}+D_{3}-D_{2}^{\prime}(\bar{v}-\bar{w})
\end{array}\right]
$$

i.e.

$$
D_{1}+D_{3}-D_{1}^{\prime}(\bar{u}-\bar{w}) \geq 0, \quad D_{2}+D_{3}-D_{2}^{\prime}(\bar{v}-\bar{w}) \geq 0, \quad \text { and } \quad \operatorname{det}(A) \geq 0
$$

The first two inequalities are true due to the relations:

$$
-1 \leq \bar{u}-\bar{w} \leq 1, \quad-1 \leq \bar{v}-\bar{w} \leq 1, \quad D_{1}>D_{2}>D_{3}>0
$$

The condition

$$
(7-4 \sqrt{3}) D_{2} \leq D_{1} \leq(7+4 \sqrt{3}) D_{2}
$$

implies $\operatorname{det}(A) \geq 0$ for all admissible $\bar{u}, \bar{w}$.

\section{Iterative methods}

Recall that

$$
\begin{aligned}
& D_{1}^{\prime} u_{x}+D_{2}^{\prime} v_{x}=D_{1} u_{x}+D_{2} v_{x}+D_{3} w_{x}, \\
& D_{1}^{\prime} u_{x x}+D_{2}^{\prime} v_{x x}=D_{1} u_{x x}+D_{2} v_{x x}+D_{3} w_{x x} .
\end{aligned}
$$

Assume that $\left(u^{(0)}, v^{(0)}, w^{(0)}\right)$ coincides with $\left(u_{0}, v_{0}, w_{0}\right)$ at $t=0$ and formulate an iterative method for (1.1)-(1.3):

$$
\begin{aligned}
& u_{t}^{(k+1)}=D_{1} u_{x x}^{(k+1)}-\left(u^{(k)}\left[D_{1} u_{x}^{(k+1)}+D_{2} v_{x}^{(k+1)}+D_{3} w_{x}^{(k+1)}\right]\right)_{x} \\
& v_{t}^{(k+1)}=D_{2} v_{x x}^{(k+1)}-\left(v^{(k)}\left[D_{1} u_{x}^{(k+1)}+D_{2} v_{x}^{(k+1)}+D_{3} w_{x}^{(k+1)}\right]\right)_{x} \\
& w_{t}^{(k+1)}=D_{3} w_{x x}^{(k+1)}-\left(w^{(k)}\left[D_{1} u_{x}^{(k+1)}+D_{2} v_{x}^{(k+1)}+D_{3} w_{x}^{(k+1)}\right]\right)_{x}
\end{aligned}
$$


with the initial condition

$$
u^{(k+1)}(0, x)=u_{0}(x), \quad v^{(k+1)}(0, x)=v_{0}(x), \quad w^{(k+1)}(0, x)=w_{0}(x)
$$

for $x \in[-L, L]$ and the Neumann boundary condition. Moreover, assume that

$$
u_{0}(x)+v_{0}(x)+w_{0}(x)=1
$$

for $x \in[-L, L]$. Denote

$$
\Delta u^{(k)}=u^{(k+1)}-u^{(k)}, \quad \Delta v^{(k)}=v^{(k+1)}-v^{(k)}, \quad \Delta w^{(k)}=w^{(k+1)}-w^{(k)} .
$$

Lemma 3.1 Assume $u_{0}, v_{0}, w_{0} \in \mathcal{X},\left(u^{(0)}, v^{(0)}, w^{(0)}\right)=\left(u_{0}, v_{0}, w_{0}\right)$ at $t=0$ and $u^{(0)}+v^{(0)}+$ $w^{(0)}=1$. If $\left(u^{(k)}, v^{(k)}, w^{(k)}\right)$ fulfills (3.1) with (3.2), the Neumann boundary condition and (3.3), then $u^{(k)}+v^{(k)}+w^{(k)}=1$.

Proof It suffices to show $u^{(k)}+v^{(k)}+w^{(k)}=1 \Rightarrow u^{(k+1)}+v^{(k+1)}+w^{(k+1)}=1$. We assume the induction hypothesis $u^{(k)}+v^{(k)}+w^{(k)}=1$. Thus

$$
\begin{aligned}
u_{t}^{(k+1)} & +v_{t}^{(k+1)}+w_{t}^{(k+1)} \\
= & D_{1} u_{x x}^{(k+1)}+D_{2} v_{x x}^{(k+1)}+D_{3} w_{x x}^{(k+1)} \\
& -\left(u_{x}^{(k)}+v_{x}^{(k)}+w_{x}^{(k)}\right)\left[D_{1} u_{x}^{(k+1)}+D_{2} v_{x}^{(k+1)}+D_{3} w_{x}^{(k+1)}\right] \\
& -\left(u^{(k)}+v^{(k)}+w^{(k)}\right)\left[D_{1} u_{x x}^{(k+1)}+D_{2} v_{x x}^{(k+1)}+D_{3} w_{x x}^{(k+1)}\right] \equiv 0 .
\end{aligned}
$$

Hence the statement is proved.

The following theorem establishes a convergence of the iterative method (3.1)-(3.2).

Theorem 3.2 Suppose $\left(u_{0}, v_{0}, w_{0}\right) \in \mathcal{X}$ and $\left(u^{(0)}, v^{(0)}, w^{(0)}\right)=\left(u_{0}, v_{0}, w_{0}\right)$ at $t=0$. If $u_{x}^{(k)}, v_{x}^{(k)}$, $w_{x}^{(k)}$ are $C^{2}$ and

$$
0 \leq u^{(k)} \leq 1, \quad 0 \leq v^{(k)} \leq 1, \quad 0 \leq w^{(k)} \leq 1 \quad \text { for } k=1,2, \ldots
$$

then the sequence $\left(u^{(k)}, v^{(k)}, w^{(k)}\right)$ defined by (3.1), (3.2) converges to the solution $(u, v, w)$ of (1.1), (1.2) in the Sobolev space $W^{1, \infty}$.

Proof As in the previous section denote the increments $\Delta u^{(k)}=u^{(k+1)}-u^{(k)}, \Delta v^{(k)}=v^{(k+1)}-$ $v^{(k)}, \Delta w^{(k)}=w^{(k+1)}-w^{(k)}$. From (3.1) we have the following differential equations:

$$
\begin{aligned}
\Delta u_{t}^{(k+1)}= & D_{1} \Delta u_{x x}^{(k+1)}-\left(u^{(k+1)}\left[D_{1}^{\prime} \Delta u_{x}^{(k+1)}+D_{2}^{\prime} \Delta v_{x}^{(k+1)}\right]\right)_{x} \\
& -\left(\Delta u^{(k)}\left[D_{1}^{\prime} u_{x}^{(k+1)}+D_{2}^{\prime} v_{x}^{(k+1)}\right]\right)_{x} \\
\Delta v_{t}^{(k+1)}= & D_{2} \Delta v_{x x}^{(k+1)}-\left(v^{(k+1)}\left[D_{1}^{\prime} \Delta u_{x}^{(k+1)}+D_{2}^{\prime} \Delta v_{x}^{(k+1)}\right]\right)_{x} \\
& -\left(\Delta v^{(k)}\left[D_{1} u_{x}^{(k+1)}+D_{2} v_{x}^{(k+1)}+D_{3} w_{x}^{(k+1)}\right]\right)_{x} .
\end{aligned}
$$


Using the Green functions $G^{1, k}, G^{2, k}$ corresponding to the differential operators

$$
\left[\begin{array}{cc}
\frac{\partial}{\partial t}-D_{1} \frac{\partial^{2}}{\partial x^{2}}+u^{(k+1)} D_{1}^{\prime} \frac{\partial^{2}}{\partial x^{2}} & D_{2}^{\prime} \frac{\partial^{2}}{\partial x^{2}} \\
D_{1}^{\prime} \frac{\partial^{2}}{\partial x^{2}} & \frac{\partial}{\partial t}-D_{2} \frac{\partial^{2}}{\partial x^{2}}+v^{(k+1)} D_{2}^{\prime} \frac{\partial^{2}}{\partial x^{2}}
\end{array}\right]
$$

we have

$$
\begin{aligned}
& {\left[\begin{array}{c}
\Delta u^{(k+1)}(t, x) \\
\Delta v^{(k+1)}(t, x)
\end{array}\right]=\int_{0}^{t} \int_{-L}^{L}\left[\begin{array}{l}
G^{1, k}(t, s, x, y) P^{1, k}(s, y) \\
G^{2, k}(t, s, x, y) P^{2, k}(s, y)
\end{array}\right] d y d s,} \\
& {\left[\begin{array}{l}
\Delta u_{x}^{(k+1)}(t, x) \\
\Delta v_{x}^{(k+1)}(t, x)
\end{array}\right]=\int_{0}^{t} \int_{-L}^{L}\left[\begin{array}{l}
G_{x}^{1, k}(t, s, x, y) P^{1, k}(s, y) \\
G_{x}^{2, k}(t, s, x, y) P^{2, k}(s, y)
\end{array}\right] d y d s,}
\end{aligned}
$$

where $P^{i, k}(s, y)$ depend on $\Delta u^{(k)}, \Delta v^{(k)}, \Delta u_{x}^{(k)}, \Delta v_{x}^{(k)}, \Delta u_{x}^{(k+1)}, \Delta v_{x}^{(k+1)}$ for $i=1,2$. The Green functions $G^{i, k}$ depend on $u^{(k)}, v^{(k)}$ and have the uniform estimates

$$
\int_{-L}^{L}\left|G^{i, k}(t, s, x, y)\right| d y \leq C, \quad \int_{-L}^{L}\left|G_{x}^{i, k}(t, s, x, y)\right| d y \leq \frac{C}{\sqrt{t-s}}
$$

with some generic constant $C$ not depending on $k$. By Lemma 3.1 there exists $M \geq 0$ such that

$$
\begin{array}{ll}
\left\|u_{x}^{(k+1)}(t, \cdot)\right\|_{L^{\infty}} \leq M, & \left\|v_{x}^{(k+1)}(t, \cdot)\right\|_{L^{\infty}} \leq M, \\
\left\|u_{x x}^{(k+1)}(t, \cdot)\right\|_{L^{\infty}} \leq M, & \left\|v_{x x}^{(k+1)}(t, \cdot)\right\|_{L^{\infty}} \leq M .
\end{array}
$$

Since

$$
\begin{aligned}
\left\|P^{1, k}(t, \cdot)\right\|_{L^{\infty}} \leq & M\left(D_{1}^{\prime}+D_{2}^{\prime}\right)\left\|\Delta u^{(k)}(t, \cdot)\right\|_{L^{\infty}}+M\left(D_{1}^{\prime}+D_{2}^{\prime}\right)\left\|\Delta u_{x}^{(k)}(t, \cdot)\right\|_{L^{\infty}} \\
& +M D_{1}^{\prime}\left\|\Delta u_{x}^{(k+1)}(t, \cdot)\right\|_{L^{\infty}}+M D_{2}^{\prime}\left\|\Delta v_{x}^{(k+1)}(t, \cdot)\right\|_{L^{\infty}}
\end{aligned}
$$

we get

$$
\begin{aligned}
\left\|\left(\Delta u^{(k+1)}, \Delta v^{(k+1)}\right)(t, \cdot)\right\|_{W^{1, \infty}}:= & \left\|\Delta u^{(k+1)}(t, \cdot)\right\|_{W^{1, \infty}}+\left\|\Delta v^{(k+1)}(t, \cdot)\right\|_{W^{1, \infty}} \\
\leq & \int_{0}^{t} \frac{2 C_{1}}{\sqrt{t-s}}\left\|\left(\Delta u^{(k)}, \Delta v^{(k)}\right)(s, \cdot)\right\|_{W^{1, \infty}} d s \\
& +\int_{0}^{t} \frac{2 C_{1}}{\sqrt{t-s}}\left\|\left(\Delta u^{(k+1)}, \Delta v^{(k+1)}\right)(s, \cdot)\right\|_{W^{1, \infty}} d s .
\end{aligned}
$$

Applying Lemma A.1 we have $\left\|\left(\Delta u^{(0)}, \Delta v^{(0)}\right)(t, \cdot)\right\|_{W^{1, \infty}} \leq K_{0}$ and by induction: $\|\left(\Delta u^{(k)}\right.$, $\left.\Delta v^{(k)}\right)(t, \cdot) \|_{W^{1, \infty}} \leq K_{k} t^{\frac{k}{2}}$ for $k=1,2, \ldots$. Hence

$$
K_{k+1}=K_{k} \frac{C}{1-2 C T^{1 / 2}} \int_{0}^{1} \frac{\theta^{k}}{\sqrt{1-\theta}} d \theta .
$$

Notice that

$$
\frac{K_{k+1}}{K_{k}}=\frac{C}{1-2 C T^{1 / 2}} \int_{0}^{1} \frac{\theta^{k}}{\sqrt{1-\theta}} d \theta \rightarrow 0 \quad \text { as } k \rightarrow \infty .
$$

By the d'Alembert's ratio test the convergence radius is $+\infty$. 
We give sufficient conditions for the successive approximations to remain in $\mathcal{X}$.

Proposition 3.3 Assume that $u_{0}, v_{0} \in C^{4}, 0<\varepsilon_{0} \leq u_{0}(x) \leq 1-\varepsilon_{0}<1,0<\varepsilon_{0} \leq v_{0}(x) \leq$ $1-\varepsilon_{0}<1$ and the sequence $\left(u^{(k)}, v^{(k)}, w^{(k)}\right)$ defined by (3.1) with the first element given by

$$
u^{(0)}(t, x)=u_{0}(x)+t k_{u}(x) \quad \text { and } \quad v^{(0)}(t, x)=v_{0}(x)+t k_{v}(x)
$$

where $k_{u}, k_{v} \in \mathcal{X}$ are of the form

$$
\begin{aligned}
& k_{u}(x)=D_{1} u_{0}^{\prime \prime}(x)-\left(u_{0}(x)\left[D_{1}^{\prime} u_{0}^{\prime}(x)+D_{2}^{\prime} v_{0}^{\prime}(x)\right]\right)_{x} \\
& k_{v}(x)=D_{2} v_{0}^{\prime \prime}(x)-\left(v_{0}(x)\left[D_{1}^{\prime} u_{0}^{\prime}(x)+D_{2}^{\prime} v_{0}^{\prime}(x)\right]\right)_{x}
\end{aligned}
$$

converges to the solution $(u, v, w)$ of $(1.1),(1.2)$ in the Sobolev space $W^{1, \infty}$. If

$$
\sum_{k=0}^{\infty} K_{k} k^{k / 2} \leq \varepsilon_{0}, \quad \text { where } K_{k}=K_{k-1} \frac{C}{1-2 C T^{1 / 2}} \int_{0}^{1} \frac{\theta^{k-1}}{\sqrt{1-\theta}} d \theta
$$

then $0 \leq u^{(k)}(t, x) \leq 1$ and $0 \leq v^{(k)}(t, x) \leq 1, k=0,1, \ldots$

Proof We have

$$
u_{t}^{(0)}(t, x)=k_{u}(x), \quad u_{x}^{(0)}(t, x)=u_{0}^{\prime}(x)+t k_{u}^{\prime}(x), \quad u_{x x}^{(0)}(t, x)=u_{0}^{\prime \prime}(x)+t k_{u}^{\prime \prime}(x) .
$$

Hence

$$
\begin{aligned}
k_{u}(x)= & D_{1} u_{0}^{\prime \prime}(x)-u_{0}^{\prime}(x)\left[D_{1}^{\prime} u_{0}^{\prime}(x)+D_{2}^{\prime} v_{0}^{\prime}(x)\right]-u_{0}(x)\left[D_{1}^{\prime} u_{0}^{\prime \prime}(x)+D_{2}^{\prime} v_{0}^{\prime \prime}(x)\right] \\
= & D_{1}\left(u_{x x}^{(0)}(t, x)-t k_{u}^{\prime \prime}(x)\right) \\
& -\left(u_{x}^{(0)}(t, x)-t k_{u}^{\prime}(x)\right)\left[D_{1}^{\prime} u_{x}^{(0)}+D_{2}^{\prime} v_{x}^{(0)}-t\left(D_{1}^{\prime} k_{u}^{\prime}(x)+D_{2}^{\prime} k_{v}^{\prime}(x)\right)\right] \\
& -\left(u^{(0)}(t, x)-t k_{u}(x)\right)\left[D_{1}^{\prime} u_{x x}^{(0)}+D_{2}^{\prime} v_{x x}^{(0)}-t\left(D_{1}^{\prime} k_{u}^{\prime \prime}(x)+D_{2}^{\prime} k_{v}^{\prime \prime}(x)\right)\right] .
\end{aligned}
$$

Thus we get

$$
\begin{aligned}
\Delta u_{t}^{(0)}(t, x)= & u_{t}^{(1)}(t, x)-u_{t}^{(0)}(t, x) \\
= & D_{1} u_{x x}^{(1)}(t, x)-u_{x}^{(0)}(t, x)\left[D_{1}^{\prime} u_{x}^{(1)}(t, x)+D_{2}^{\prime} v_{x}^{(1)}(t, x)\right] \\
& -u^{(0)}(t, x)\left[D_{1}^{\prime} u_{x x}^{(1)}(t, x)+D_{2}^{\prime} v_{x x}^{(1)}(t, x)\right]-k_{u}(x) \\
= & D_{1} \Delta u_{x x}^{(0)}(t, x)-\left(u^{(0)}(t, x)\left[D_{1}^{\prime} \Delta u_{x}^{(0)}(t, x)+D_{2}^{\prime} \Delta v_{x}^{(0)}(t, x)\right]\right)_{x} \\
& +t R_{1}(x)+t^{2} R_{2}(x),
\end{aligned}
$$

where

$$
\begin{aligned}
R_{1}(x):= & D_{1} k_{u}^{\prime \prime}(x)-u_{x}^{(0)}(t, x)\left(D_{1}^{\prime} k_{u}^{\prime}(x)+D_{2}^{\prime} k_{v}^{\prime}(x)\right)-k_{u}^{\prime}(x)\left[D_{1}^{\prime} u_{x}^{(0)}(t, x)+D_{2}^{\prime} v_{x}^{(0)}(t, x)\right] \\
& -u^{(0)}(t, x)\left(D_{1}^{\prime} k_{u}^{\prime \prime}(x)+D_{2}^{\prime} k_{v}^{\prime \prime}(x)\right)-k_{u}(x)\left[D_{1}^{\prime} u_{x x}^{(0)}(t, x)+D_{2}^{\prime} v_{x x}^{(0)}(t, x)\right], \\
R_{2}(x):= & k_{u}^{\prime}(x)\left(D_{1}^{\prime} k_{u}^{\prime}(x)+D_{2}^{\prime} k_{v}^{\prime}(x)\right)+k_{u}(x)\left(D_{1}^{\prime} k_{u}^{\prime \prime}(x)+D_{2}^{\prime} k_{v}^{\prime \prime}(x)\right) .
\end{aligned}
$$


For $0 \leq t \leq T$ we have

$$
\|R\|_{L^{\infty}}=\left\|t R_{1}+t^{2} R_{2}\right\|_{L^{\infty}} \leq T\left(\left\|R_{1}\right\|_{L^{\infty}}+T\left\|R_{2}\right\|_{L^{\infty}}\right)=: K_{0} .
$$

Thus

$$
\left\|\left(\Delta u^{(0)}, \Delta v^{(0)}\right)(t, \cdot)\right\|_{W^{1, \infty}} \leq \int_{0}^{t} \frac{C}{\sqrt{t-s}}\left[\left\|\left(\Delta u^{(0)}, \Delta v^{(0)}\right)(s, \cdot)\right\|_{W^{1, \infty}}+K_{0}\right] d s .
$$

Applying Lemma A.1 we have

$$
\left\|\left(\Delta u^{(0)}, \Delta v^{(0)}\right)(t, \cdot)\right\|_{W^{1, \infty}} \leq K_{1} t^{1 / 2}
$$

and by induction $\left\|\left(\Delta u^{(k)}, \Delta v^{(k)}\right)(t, \cdot)\right\|_{W^{1, \infty}} \leq K_{k+1} t^{\frac{k+1}{2}}$ for $k=1,2, \ldots$ Hence

$$
K_{k+1}=K_{k} \frac{C}{1-2 C T^{1 / 2}} \int_{0}^{1} \frac{\theta^{k}}{\sqrt{1-\theta}} d \theta
$$

Remark 3.4 The functions $k_{u}, k_{v} \in \mathcal{X}$ can be slightly perturbed near the lateral boundary in order to fulfill the Neumann boundary condition.

\section{Convergence of the Newton method}

As in the previous section denote

$$
\Delta u^{(k)}=u^{(k+1)}-u^{(k)}, \quad \Delta v^{(k)}=v^{(k+1)}-v^{(k)}, \quad \Delta w^{(k)}=w^{(k+1)}-w^{(k)} .
$$

We assume that $\left(u^{(0)}, v^{(0)}, w^{(0)}\right)=\left(u_{0}, v_{0}, w_{0}\right)$ at $t=0$ and formulate the Newton method for (1.1)-(1.3):

$$
\begin{aligned}
u_{t}^{(k+1)}= & D_{1} u_{x x}^{(k+1)}-\left(u^{(k)}\left[D_{1}^{\prime} u_{x}^{(k)}+D_{2}^{\prime} v_{x}^{(k)}\right]\right)_{x} \\
& -\left(\Delta u^{(k)}\left[D_{1}^{\prime} u_{x}^{(k)}+D_{2}^{\prime} v_{x}^{(k)}\right]\right)_{x}-\left(u^{(k)}\left[D_{1}^{\prime} \Delta u_{x}^{(k)}+D_{2}^{\prime} \Delta v_{x}^{(k)}\right]\right)_{x} \\
v_{t}^{(k+1)}= & D_{2} v_{x x}^{(k+1)}-\left(v^{(k)}\left[D_{1}^{\prime} u_{x}^{(k)}+D_{2}^{\prime} v_{x}^{(k)}\right]\right)_{x} \\
& -\left(\Delta v^{(k)}\left[D_{1}^{\prime} u_{x}^{(k)}+D_{2}^{\prime} v_{x}^{(k)}\right]\right)_{x}-\left(v^{(k)}\left[D_{1}^{\prime} \Delta u_{x}^{(k)}+D_{2}^{\prime} \Delta v_{x}^{(k)}\right]\right)_{x} \\
w_{t}^{(k+1)}= & D_{3} w_{x x}^{(k+1)}-\left(w^{(k)}\left[D_{1}^{\prime} u_{x}^{(k)}+D_{2}^{\prime} v_{x}^{(k)}\right]\right)_{x} \\
& -\left(\Delta w^{(k)}\left[D_{1}^{\prime} u_{x}^{(k)}+D_{2}^{\prime} v_{x}^{(k)}\right]\right)_{x}-\left(w^{(k)}\left[D_{1}^{\prime} \Delta u_{x}^{(k)}+D_{2}^{\prime} \Delta v_{x}^{(k)}\right]\right)_{x}
\end{aligned}
$$

with the initial condition (3.2) and the Neumann boundary condition.

Lemma 4.1 Assume $u_{0}, v_{0}, w_{0} \in \mathcal{X},\left(u^{(0)}, v^{(0)}, w^{(0)}\right)=\left(u_{0}, v_{0}, w_{0}\right)$ at $t=0$ and $u^{(0)}+v^{(0)}+$ $w^{(0)}=1$. If $\left(u^{(k)}, v^{(k)}, w^{(k)}\right)$ fulfills (4.1) with (3.2) and the Neumann boundary condition, then $u^{(k)}+v^{(k)}+w^{(k)}=1$. 
Proof We show $u^{(k)}+v^{(k)}+w^{(k)}=1 \Rightarrow u^{(k+1)}+v^{(k+1)}+w^{(k+1)}=1$. The only solution to the differential equation

$$
\begin{aligned}
u_{t}^{(k+1)} & +v_{t}^{(k+1)}+w_{t}^{(k+1)} \\
= & -\left(u_{x}^{(k+1)}+v_{x}^{(k+1)}+w_{x}^{(k+1)}\right)\left[D_{1} u_{x}^{(k)}+D_{2} v_{x}^{(k)}+D_{3} w_{x}^{(k)}\right] \\
& -\left(u^{(k+1)}+v^{(k+1)}+w^{(k+1)}-1\right)\left[D_{1} u_{x x}^{(k)}+D_{2} v_{x x}^{(k)}+D_{3} w_{x x}^{(k)}\right]
\end{aligned}
$$

is $u^{(k+1)}+v^{(k+1)}+w^{(k+1)} \equiv 1$.

The following theorem establishes the convergence of the Newton method.

Theorem 4.2 Suppose $\left(u_{0}, v_{0}, w_{0}\right) \in \mathcal{X}$ and $\left(u^{(0)}, v^{(0)}, w^{(0)}\right)=\left(u_{0}, v_{0}, w_{0}\right)$ at $t=0$. If $u_{x}^{(k)}, v_{x}^{(k)}$, $w_{x}^{(k)}$ are $C^{2}$ and

$$
0 \leq u^{(k)} \leq 1, \quad 0 \leq v^{(k)} \leq 1, \quad 0 \leq w^{(k)} \leq 1 \quad \text { for } k=1,2, \ldots
$$

then the sequence $\left(u^{(k)}, v^{(k)}, w^{(k)}\right)$ defined by (4.1), (3.2) converges to the solution $(u, v, w)$ of (1.1)-(1.3) with respect to the norms in the Sobolev space $W^{1, \infty}$.

Proof We have the following differential equations:

$$
\begin{aligned}
\Delta u_{t}^{(k+1)}= & D_{1} \Delta u_{x x}^{(k+1)}-\left(\Delta u^{(k)}\left[D_{1}^{\prime} \Delta u_{x}^{(k)}+D_{2}^{\prime} \Delta v_{x}^{(k)}\right]\right)_{x} \\
& -\left(\Delta u^{(k+1)}\left[D_{1}^{\prime} u_{x}^{(k+1)}+D_{2}^{\prime} v_{x}^{(k+1)}\right]\right)_{x}-\left(u^{(k+1)}\left[D_{1}^{\prime} \Delta u_{x}^{(k+1)}+D_{2}^{\prime} \Delta v_{x}^{(k+1)}\right]\right)_{x} \\
\Delta v_{t}^{(k+1)}= & D_{2} \Delta v_{x x}^{(k+1)}-\left(\Delta v^{(k)}\left[D_{1}^{\prime} \Delta u_{x}^{(k)}+D_{2}^{\prime} \Delta v_{x}^{(k)}\right]\right)_{x} \\
& -\left(\Delta v^{(k+1)}\left[D_{1}^{\prime} u_{x}^{(k+1)}+D_{2}^{\prime} v_{x}^{(k+1)}\right]\right)_{x}-\left(v^{(k+1)}\left[D_{1}^{\prime} \Delta u_{x}^{(k+1)}+D_{2}^{\prime} \Delta v_{x}^{(k+1)}\right]\right)_{x} \\
\Delta w_{t}^{(k+1)}= & D_{3} \Delta w_{x x}^{(k+1)}-\left(\Delta w^{(k)}\left[D_{1}^{\prime} \Delta u_{x}^{(k)}+D_{2}^{\prime} \Delta v_{x}^{(k)}\right]\right)_{x} \\
& -\left(\Delta w^{(k+1)}\left[D_{1}^{\prime} u_{x}^{(k+1)}+D_{2}^{\prime} v_{x}^{(k+1)}\right]\right)_{x}-\left(w^{(k+1)}\left[D_{1}^{\prime} \Delta u_{x}^{(k+1)}+D_{2}^{\prime} \Delta v_{x}^{(k+1)}\right]\right)_{x} .
\end{aligned}
$$

By the Green functions $G^{1, k}, G^{2, k}$ :

$$
\begin{aligned}
\Delta u^{(k+1)}(t, x)= & \int_{0}^{t} \int_{-L}^{L} G^{1, k}(t, s, x, y)\left(\Delta u^{(k)}(s, y)\left[D_{1}^{\prime} \Delta u_{y}^{(k)}(s, y)+D_{2}^{\prime} \Delta v_{y}^{(k)}(s, y)\right]\right)_{y} d y d s \\
& +\int_{0}^{t} \int_{-L}^{L} G^{1, k}(t, s, x, y) \Delta u_{y}^{(k+1)}(s, y)\left[D_{1}^{\prime} u_{y}^{(k+1)}(s, y)+D_{2}^{\prime} v_{y}^{(k+1)}(s, y)\right] d y d s \\
& +\int_{0}^{t} \int_{-L}^{L} G^{1, k}(t, s, x, y) \Delta u^{(k+1)}(s, y)\left[D_{1}^{\prime} u_{y y}^{(k+1)}(s, y)+D_{2}^{\prime} v_{y y}^{(k+1)}(s, y)\right] d y d s \\
& +\int_{0}^{t} \int_{-L}^{L} G^{1, k}(t, s, x, y) u_{y}^{(k+1)}(s, y)\left[D_{1}^{\prime} \Delta u_{y}^{(k+1)}(s, y)+D_{2}^{\prime} \Delta v_{y}^{(k+1)}(s, y)\right] d y d s
\end{aligned}
$$

Using the integration by parts we get

$$
\begin{aligned}
& \int_{0}^{t} \int_{-L}^{L} G^{1, k}(t, s, x, y)\left(\Delta u^{(k)}(s, y)\left[D_{1}^{\prime} \Delta u_{y}^{(k)}(s, y)+D_{2}^{\prime} \Delta v_{y}^{(k)}(s, y)\right]\right)_{y} d y d s \\
& \quad=-\int_{0}^{t} \int_{-L}^{L} G_{y}^{1, k}(t, s, x, y) \Delta u^{(k)}(s, y)\left[D_{1}^{\prime} \Delta u_{y}^{(k)}(s, y)+D_{2}^{\prime} \Delta v_{y}^{(k)}(s, y)\right] d y d s .
\end{aligned}
$$


From the following property:

$$
\int_{-L}^{L}\left|G_{y}^{i, k}(t, s, x, y)\right| d y \leq \frac{C}{\sqrt{t-s}}
$$

estimates like (3.5), (3.6), and $x y \leq \frac{1}{2}\left(x^{2}+y^{2}\right)$ we obtain

$$
\left\|\Delta u^{(k+1)}(t, \cdot)\right\|_{L^{\infty}} \leq \int_{0}^{t} \frac{C_{2}}{2 \sqrt{t-s}} Q^{1, k}(s) d s,
$$

where

$$
\begin{aligned}
Q^{1, k}(s)= & \left\|\Delta u^{(k)}(s, \cdot)\right\|_{L^{\infty}}^{2}+\left\|\Delta u_{y}^{(k)}(s, \cdot)\right\|_{L^{\infty}}^{2}+\left\|\Delta v_{y}^{(k)}(s, \cdot)\right\|_{L^{\infty}}^{2}+\left\|\Delta u_{y}^{(k+1)}(s, \cdot)\right\|_{L^{\infty}} \\
& +\left\|\Delta u^{(k+1)}(s, \cdot)\right\|_{L^{\infty}} d s+\left\|\Delta u_{y}^{(k+1)}(s, \cdot)\right\|_{L^{\infty}}+\left\|\Delta v_{y}^{(k+1)}(s, \cdot)\right\|_{L^{\infty}} .
\end{aligned}
$$

Similarly

$$
\left\|\Delta v^{(k+1)}(t, \cdot)\right\|_{L^{\infty}} \leq \int_{0}^{t} \frac{C_{2}}{2 \sqrt{t-s}} Q^{2, k}(s) d s,
$$

where

$$
\begin{aligned}
Q^{2, k}(s)= & \left\|\Delta v^{(k)}(s, \cdot)\right\|_{L^{\infty}}^{2}+\left\|\Delta u_{y}^{(k)}(s, \cdot)\right\|_{L^{\infty}}^{2}+\left\|\Delta v_{y}^{(k)}(s, \cdot)\right\|_{L^{\infty}}^{2}+\left\|\Delta v_{y}^{(k+1)}(s, \cdot)\right\|_{L^{\infty}} \\
& +\left\|\Delta v^{(k+1)}(s, \cdot)\right\|_{L^{\infty}}+\left\|\Delta u_{y}^{(k+1)}(s, \cdot)\right\|_{L^{\infty}}+\left\|\Delta v_{y}^{(k+1)}(s, \cdot)\right\|_{L^{\infty}} .
\end{aligned}
$$

We have

$$
\begin{aligned}
& \Delta u_{x}^{(k+1)}(t, x) \\
& =\int_{0}^{t} \int_{-L}^{L} G_{x}^{1, k}(t, s, x, y)\left(\Delta u^{(k)}(s, y)\left[D_{1}^{\prime} \Delta u_{y}^{(k)}(s, y)+D_{2}^{\prime} \Delta v_{y}^{(k)}(s, y)\right]\right)_{y} d y d s \\
& \quad+\int_{0}^{t} \int_{-L}^{L} G_{x}^{1, k}(t, s, x, y) \Delta u_{y}^{(k+1)}(s, y)\left[D_{1}^{\prime} u_{y}^{(k+1)}(s, y)+D_{2}^{\prime} v_{y}^{(k+1)}(s, y)\right] d y d s \\
& \quad+\int_{0}^{t} \int_{-L}^{L} G_{x}^{1, k}(t, s, x, y) \Delta u^{(k+1)}(s, y)\left[D_{1}^{\prime} u_{y y}^{(k+1)}(s, y)+D_{2}^{\prime} v_{y y}^{(k+1)}(s, y)\right] d y d s \\
& \quad+\int_{0}^{t} \int_{-L}^{L} G_{x}^{1, k}(t, s, x, y) u_{y}^{(k+1)}(s, y)\left[D_{1}^{\prime} \Delta u_{y}^{(k+1)}(s, y)+D_{2}^{\prime} \Delta v_{y}^{(k+1)}(s, y)\right] d y d s .
\end{aligned}
$$

By integration by parts:

$$
\begin{aligned}
& \int_{0}^{t} \int_{-L}^{L} G_{x}^{1, k}(t, s, x, y)\left(\Delta u^{(k)}(s, y)\left[D_{1}^{\prime} \Delta u_{y}^{(k)}(s, y)+D_{2}^{\prime} \Delta v_{y}^{(k)}(s, y)\right]\right)_{y} d y d s \\
& \quad=-\int_{0}^{t} \int_{-L}^{L} G_{x y}^{1, k}(t, s, x, y) \Delta u^{(k)}(s, y)\left[D_{1}^{\prime} \Delta u_{y}^{(k)}(s, y)+D_{2}^{\prime} \Delta v_{y}^{(k)}(s, y)\right] d y d s .
\end{aligned}
$$


Since $\Delta u^{(k)}(s, y), \Delta u_{y}^{(k)}(s, y), \Delta v_{y}^{(k)}(s, y)$ satisfy the Lipschitz condition, we have the estimates (see [12])

$$
\begin{aligned}
& \int_{-L}^{L}\left|G_{x y}^{i, k}(t, s, x, y) \Delta u^{(k)}(s, y) \Delta u_{y}^{(k)}(s, y)\right| d y \\
& \quad \leq \int_{0}^{t} \frac{C_{3}}{(t-s)^{3 / 4}}\left\|\Delta u^{(k)}(s, \cdot)\right\|_{L^{\infty}}\left\|\Delta u_{y}^{(k)}(s, \cdot)\right\|_{L^{\infty}} d s
\end{aligned}
$$

and

$$
\begin{aligned}
& \int_{-L}^{L}\left|G_{x y}^{i, k}(t, s, x, y) \Delta u^{(k)}(s, y) \Delta v_{y}^{(k)}(s, y)\right| d y \\
& \quad \leq \int_{0}^{t} \frac{C_{3}}{(t-s)^{3 / 4}}\left\|\Delta u^{(k)}(s, \cdot)\right\|_{L^{\infty}}\left\|\Delta v_{y}^{(k)}(s, \cdot)\right\|_{L^{\infty}} d s .
\end{aligned}
$$

Hence

$$
\begin{aligned}
& \left\|\Delta u_{x}^{(k+1)}(t, \cdot)\right\|_{L^{\infty}} \\
& \leq \int_{0}^{t} \frac{C_{4}}{(t-s)^{3 / 4}}\left(\left\|\Delta u^{(k)}(s, \cdot)\right\|_{L^{\infty}}^{2}+\left\|\Delta u_{y}^{(k)}(s, \cdot)\right\|_{L^{\infty}}^{2}+\left\|\Delta v_{y}^{(k)}(s, \cdot)\right\|_{L^{\infty}}^{2}\right) d s \\
& \quad+\int_{0}^{t} \frac{C_{5}}{\sqrt{t-s}}\left(\left\|\Delta u_{y}^{(k+1)}(s, \cdot)\right\|_{L^{\infty}}+\left\|\Delta u^{(k+1)}(s, \cdot)\right\|_{L^{\infty}}+\left\|\Delta u_{y}^{(k+1)}(s, \cdot)\right\|_{L^{\infty}}\right. \\
& \left.\quad+\left\|\Delta v_{y}^{(k+1)}(s, \cdot)\right\|_{L^{\infty}}\right) d s .
\end{aligned}
$$

Similarly

$$
\begin{aligned}
& \left\|\Delta v_{x}^{(k+1)}(t, \cdot)\right\|_{L^{\infty}} \\
& \leq \int_{0}^{t} \frac{C_{4}}{(t-s)^{3 / 4}}\left(\left\|\Delta v^{(k)}(s, \cdot)\right\|_{L^{\infty}}^{2}+\left\|\Delta u_{y}^{(k)}(s, \cdot)\right\|_{L^{\infty}}^{2}+\left\|\Delta v_{y}^{(k)}(s, \cdot)\right\|_{L^{\infty}}^{2}\right) d s \\
& \quad+\int_{0}^{t} \frac{C_{5}}{\sqrt{t-s}}\left(\left\|\Delta v_{y}^{(k+1)}(s, \cdot)\right\|_{L^{\infty}}+\left\|\Delta v^{(k+1)}(s, \cdot)\right\|_{L^{\infty}}+\left\|\Delta u_{y}^{(k+1)}(s, \cdot)\right\|_{L^{\infty}}\right. \\
& \left.\quad+\left\|\Delta v_{y}^{(k+1)}(s, \cdot)\right\|_{L^{\infty}}\right) d s .
\end{aligned}
$$

We have

$$
\begin{aligned}
\left\|\left(\Delta u^{(k+1)}, \Delta v^{(k+1)}\right)(t, \cdot)\right\|_{W^{1, \infty}} \leq & \int_{0}^{t} \frac{C_{4}}{(t-s)^{3 / 4}}\left\|\left(\Delta u^{(k)}, \Delta v^{(k)}\right)(s, \cdot)\right\|_{W^{1, \infty}}^{2} d s \\
& +\int_{0}^{t} \frac{C_{5}}{\sqrt{t-s}}\left\|\left(\Delta u^{(k+1)}, \Delta v^{(k+1)}\right)(s, \cdot)\right\|_{W^{1, \infty}} d s .
\end{aligned}
$$

We apply Lemma A.1:

$$
K_{k+1} t^{r_{k+1}}\left(1-2 C_{5} T^{1 / 2}\right) \geq C_{4} K_{k} t^{2 r_{k+1}+3 / 4} \int_{0}^{1} \frac{\theta^{2 r_{k}}}{(1-\theta)^{3 / 4}} d \theta,
$$

where

$$
r_{k}=\frac{3}{4}\left(2^{k}-1\right) \approx 2^{k} \quad \text { and } \quad K_{k} \approx A^{2^{k}} .
$$


We give sufficient conditions for the successive approximations to remain in $\mathcal{X}$.

Proposition 4.3 Assume that $u_{0}, v_{0} \in C^{4}, 0<\varepsilon_{0} \leq u_{0}(x) \leq 1-\varepsilon_{0}<1,0<\varepsilon_{0} \leq v_{0}(x) \leq$ $1-\varepsilon_{0}<1$ and the sequence $\left(u^{(k)}, v^{(k)}, w^{(k)}\right)$ defined by (4.1) with the first element given by

$$
u^{(0)}(t, x)=u_{0}(x)+t k_{u}(x) \quad \text { and } \quad v^{(0)}(t, x)=v_{0}(x)+t k_{\nu}(x)
$$

where $k_{u}, k_{v} \in \mathcal{X}$ are of the form

$$
\begin{aligned}
& k_{u}(x)=D_{1} u_{0}^{\prime \prime}(x)-\left(u_{0}(x)\left[D_{1}^{\prime} u_{0}^{\prime}(x)+D_{2}^{\prime} v_{0}^{\prime}(x)\right]\right)_{x^{\prime}} \\
& k_{v}(x)=D_{2} v_{0}^{\prime \prime}(x)-\left(v_{0}(x)\left[D_{1}^{\prime} u_{0}^{\prime}(x)+D_{2}^{\prime} v_{0}^{\prime}(x)\right]\right)_{x}
\end{aligned}
$$

converges to the solution $(u, v, w)$ of $(1.1),(1.2)$ in the Sobolev space $W^{1, \infty}$. If

$$
\sum_{k=0}^{\infty} \tilde{K}_{k} t^{\frac{3 k}{4}} \leq \varepsilon_{0}, \quad \tilde{K}_{k}:=\tilde{K}_{k-1} \frac{\tilde{C}}{1-2 C T^{1 / 2}} \int_{0}^{1} \frac{\theta^{k-1}}{(1-\theta)^{3 / 4}} d \theta,
$$

then $0 \leq u^{(k)}(t, x) \leq 1$ and $0 \leq v^{(k)}(t, x) \leq 1, k=0,1, \ldots$

Proof We have

$$
\begin{aligned}
\Delta u_{t}^{(0)}(t, x)= & u_{t}^{(1)}(t, x)-u_{t}^{(0)}(t, x) \\
= & D_{1} u_{x x}^{(1)}-\left(u^{(0)}\left[D_{1}^{\prime} u_{x}^{(0)}+D_{2}^{\prime} v_{x}^{(0)}\right]\right)_{x} \\
& -\left(\Delta u^{(0)}\left[D_{1}^{\prime} u_{x}^{(0)}+D_{2}^{\prime} v_{x}^{(0)}\right]\right)_{x}-\left(u^{(0)}\left[D_{1}^{\prime} \Delta u_{x}^{(0)}+D_{2}^{\prime} \Delta v_{x}^{(0)}\right]\right)_{x}-k_{u}(x) \\
= & D_{1} \Delta u_{x x}^{(0)}-\left(\Delta u^{(0)}\left[D_{1}^{\prime} u_{x}^{(0)}+D_{2}^{\prime} v_{x}^{(0)}\right]\right)_{x}-\left(u^{(0)}\left[D_{1}^{\prime} \Delta u_{x}^{(0)}+D_{2}^{\prime} \Delta v_{x}^{(0)}\right]\right)_{x} \\
& +t R_{1}(x)+t^{2} R_{2}(x),
\end{aligned}
$$

where $R_{1}(x)$ and $R_{2}(x)$ are of the same form as in the proof of Proposition 3.3. Since

$$
\left\|t R_{1}+t^{2} R_{2}\right\|_{L^{\infty}} \leq T\left(\left\|R_{1}\right\|_{L^{\infty}}+T\left\|R_{2}\right\|_{L^{\infty}}\right)=: \tilde{K}_{0}
$$

we have

$$
\begin{aligned}
& \left\|\left(\Delta u^{(0)}, \Delta v^{(0)}\right)(t, \cdot)\right\|_{W^{1, \infty}} \\
& \quad \leq \int_{0}^{t} \frac{C}{\sqrt{t-s}}\left\|\left(\Delta u^{(0)}, \Delta v^{(0)}\right)(s, \cdot)\right\|_{W^{1, \infty}} d s+\int_{0}^{t} \frac{\tilde{C}}{(t-s)^{3 / 4}} \tilde{K}_{0} d s .
\end{aligned}
$$

Applying Lemma A.1 we get

$$
\left\|\left(\Delta u^{(0)}, \Delta v^{(0)}\right)(t, \cdot)\right\|_{W^{1, \infty}} \leq \tilde{K}_{1} t^{3 / 4}
$$

and by induction $\left\|\left(\Delta u^{(k)}, \Delta v^{(k)}\right)(t, \cdot)\right\|_{W^{1, \infty}} \leq \tilde{K}_{k+1} t^{\frac{3(k+1)}{4}}$ for $k=1,2, \ldots$ Hence

$$
\tilde{K}_{k+1}:=\tilde{K}_{k} \frac{\tilde{C}}{1-2 C T^{1 / 2}} \int_{0}^{1} \frac{\theta^{k}}{(1-\theta)^{3 / 4}} d \theta .
$$




\section{Conclusions}

Assume that $\left(u^{(0)}, v^{(0)}, w^{(0)}\right)$ coincides with $\left(u_{0}, v_{0}, w_{0}\right)$ at $t=0$ and consider the following iterative scheme for (1.1)-(1.3):

$$
\begin{aligned}
& u_{t}^{(k+1)}=D_{1} u_{x x}^{(k+1)}-\left(u^{(k+1)}\left[D_{1} u_{x}^{(k)}+D_{2} v_{x}^{(k)}+D_{3} w_{x}^{(k)}\right]\right)_{x}, \\
& v_{t}^{(k+1)}=D_{2} v_{x x}^{(k+1)}-\left(v^{(k+1)}\left[D_{1} u_{x}^{(k)}+D_{2} v_{x}^{(k)}+D_{3} w_{x}^{(k)}\right]\right)_{x}, \\
& w_{t}^{(k+1)}=D_{3} w_{x x}^{(k+1)}-\left(w^{(k+1)}\left[D_{1} u_{x}^{(k)}+D_{2} v_{x}^{(k)}+D_{3} w_{x}^{(k)}\right]\right)_{x}
\end{aligned}
$$

with the initial condition

$$
u^{(k+1)}(0, x)=u_{0}(x), \quad v^{(k+1)}(0, x)=v_{0}(x), \quad w^{(k+1)}(0, x)=w_{0}(x)
$$

for $x \in[-L, L]$ and the Neumann boundary condition. Denote

$$
\Delta u^{(k)}=u^{(k+1)}-u^{(k)}, \quad \Delta v^{(k)}=v^{(k+1)}-v^{(k)}, \quad \Delta w^{(k)}=w^{(k+1)}-w^{(k)} .
$$

Convergence problems occur in $L^{2}$ and the Sobolev norm $W^{1, \infty}$. Our attempt to obtain the following relation for the increments $\Delta u^{(k)}, \Delta v^{(k)}, \Delta w^{(k)}$ :

$$
\frac{d}{d t} A_{k+1}(t) \leq C\left[A_{k+1}(t)+A_{k}(t)\right], \quad A_{k}(t)=\int_{-L}^{L}\left[\left(\Delta u^{(k)}\right)^{2}+\left(\Delta v^{(k)}\right)^{2}+\left(\Delta w^{(k)}\right)^{2}\right] d x
$$

was unsuccessful as it is difficult to estimate the following component:

$$
\int_{-L}^{L} \Delta u^{(k+1)}\left(u^{(k+1)}\left[D_{1} \Delta u_{x}^{(k)}+D_{2} \Delta v_{x}^{(k)}+D_{3} \Delta w_{x}^{(k)}\right]\right)_{x} d x
$$

This example of iterations shows that strongly coupled systems cause serious problems with their approximation. We think that the ternary system and suitable approximations to it will be somehow expressed in an abstract way, based on a Conti-Opial type theorem, like in [13].

In order to illustrate fast convergence of Newton's iterations we provide numerical examples with $D_{1}=1, D_{2}=0.5, D_{3}=0.2$, and $u_{0}, v_{0}$ being sample piecewise polynomial functions taking values in $[0.2,0.8]$. We check the differences $u^{k+1}-u^{k}$ and $v^{k+1}-v^{k}$ for $k=0,1,2,3,4$. Our computer programs are performed by implicit finite difference methods with steps $h_{0}=h_{1}=0.01$; see Table 1 (direct iterations), Table 2 (Newton's iterations).

\section{Appendix}

Lemma A.1 Assume that

$$
z(t) \leq \int_{0}^{t} \frac{C}{\sqrt{t-s}} z(s) d s+\int_{0}^{t} \frac{\tilde{C}}{(t-s)^{\alpha}} p(s) d s \quad \text { and } \quad p(s) \leq K s^{m}
$$

Then $z(t) \leq \tilde{K} t^{m+\alpha}$ for $t \in[0, T]$, where $\frac{1}{2} \leq \alpha<1$ and $1-2 C T^{1 / 2}>0$. 
Table 1 Maximal differences between successive approximations $u^{(k)}$ by direct iterations (3.1) with $D_{1}=1, D_{2}=0.5, D_{3}=0.2, h=0.01, h_{0}=0.01$

\begin{tabular}{llllll}
\hline $\boldsymbol{t}$ & $\left|\boldsymbol{u}^{(\mathbf{1})}-\boldsymbol{u}^{(\mathbf{0})}\right|$ & $\left|\boldsymbol{u}^{(\mathbf{2})}-\boldsymbol{u}^{(\mathbf{1})}\right|$ & $\left|\boldsymbol{u}^{(\mathbf{3})}-\boldsymbol{u}^{(\mathbf{2})}\right|$ & $\left|\boldsymbol{u}^{(\mathbf{4})}-\boldsymbol{u}^{\mathbf{( 3 )}}\right|$ & $\left|\boldsymbol{u}^{(\mathbf{5 )}}-\boldsymbol{u}^{(\mathbf{4})}\right|$ \\
\hline 0.00 & $0.000000 \mathrm{e}+00$ & $0.000000 \mathrm{e}+00$ & $0.000000 \mathrm{e}+00$ & $0.000000 \mathrm{e}+00$ & $0.000000 \mathrm{e}+00$ \\
0.05 & $1.023328 \mathrm{e}-01$ & $3.742852 \mathrm{e}-03$ & $1.383520 \mathrm{e}-04$ & $7.409068 \mathrm{e}-06$ & $3.256123 \mathrm{e}-07$ \\
0.10 & $1.476173 \mathrm{e}-01$ & $6.990620 \mathrm{e}-03$ & $2.908263 \mathrm{e}-04$ & $1.835346 \mathrm{e}-05$ & $1.004908 \mathrm{e}-06$ \\
0.15 & $1.783626 \mathrm{e}-01$ & $9.324930 \mathrm{e}-03$ & $4.031573 \mathrm{e}-04$ & $2.746097 \mathrm{e}-05$ & $1.675459 \mathrm{e}-06$ \\
0.20 & $2.028066 \mathrm{e}-01$ & $1.096861 \mathrm{e}-02$ & $4.788241 \mathrm{e}-04$ & $3.397007 \mathrm{e}-05$ & $2.215127 \mathrm{e}-06$ \\
0.25 & $2.239067 \mathrm{e}-01$ & $1.206830 \mathrm{e}-02$ & $5.253484 \mathrm{e}-04$ & $3.806322 \mathrm{e}-05$ & $2.601687 \mathrm{e}-06$ \\
0.30 & $2.425240 \mathrm{e}-01$ & $1.273201 \mathrm{e}-02$ & $5.499511 \mathrm{e}-04$ & $4.011546 \mathrm{e}-05$ & $2.844953 \mathrm{e}-06$ \\
0.35 & $2.593736 \mathrm{e}-01$ & $1.304597 \mathrm{e}-02$ & $5.585483 \mathrm{e}-04$ & $4.051817 \mathrm{e}-05$ & $2.967818 \mathrm{e}-06$ \\
0.40 & $2.745434 \mathrm{e}-01$ & $1.307759 \mathrm{e}-02$ & $5.556250 \mathrm{e}-04$ & $3.970391 \mathrm{e}-05$ & $2.985870 \mathrm{e}-06$ \\
\hline
\end{tabular}

Table 2 Maximal differences between successive approximations $u^{(k)}$ by Newton's method (4.1) with $D_{1}=1, D_{2}=0.5, D_{3}=0.2, h=0.01, h_{0}=0.01$

\begin{tabular}{llllll}
\hline $\boldsymbol{t}$ & $\left|\boldsymbol{u}^{(\mathbf{1})}-\boldsymbol{u}^{(\mathbf{0})}\right|$ & $\left|\boldsymbol{u}^{(\mathbf{2})}-\boldsymbol{u}^{(\mathbf{1})}\right|$ & $\left|\boldsymbol{u}^{(\mathbf{3})}-\boldsymbol{u}^{(\mathbf{2})}\right|$ & $\left|\boldsymbol{u}^{(\mathbf{4})}-\boldsymbol{u}^{(\mathbf{3})}\right|$ & $\left|\boldsymbol{u}^{(\mathbf{5 )}}-\boldsymbol{u}^{(\mathbf{4})}\right|$ \\
\hline 0.00 & $0.000000 \mathrm{e}+00$ & $0.000000 \mathrm{e}+00$ & $0.000000 \mathrm{e}+00$ & $0.000000 \mathrm{e}+00$ & $0.000000 \mathrm{e}+00$ \\
0.05 & $9.970638 \mathrm{e}-02$ & $1.703717 \mathrm{e}-03$ & $6.949251 \mathrm{e}-07$ & $1.628697 \mathrm{e}-13$ & $8.038015 \mathrm{e}-14$ \\
0.10 & $1.430019 \mathrm{e}-01$ & $3.927916 \mathrm{e}-03$ & $4.076982 \mathrm{e}-06$ & $4.423240 \mathrm{e}-12$ & $5.245804 \mathrm{e}-14$ \\
0.15 & $1.723550 \mathrm{e}-01$ & $5.877059 \mathrm{e}-03$ & $9.749392 \mathrm{e}-06$ & $2.192441 \mathrm{e}-11$ & $3.896883 \mathrm{e}-14$ \\
0.20 & $1.956575 \mathrm{e}-01$ & $7.536084 \mathrm{e}-03$ & $1.659814 \mathrm{e}-05$ & $1.148864 \mathrm{e}-10$ & $3.987088 \mathrm{e}-14$ \\
0.25 & $2.155355 \mathrm{e}-01$ & $8.989691 \mathrm{e}-03$ & $2.404146 \mathrm{e}-05$ & $4.279036 \mathrm{e}-10$ & $3.957945 \mathrm{e}-14$ \\
0.30 & $2.332049 \mathrm{e}-01$ & $1.029947 \mathrm{e}-02$ & $3.161596 \mathrm{e}-05$ & $1.167791 \mathrm{e}-09$ & $3.042011 \mathrm{e}-14$ \\
0.35 & $2.489078 \mathrm{e}-01$ & $1.153614 \mathrm{e}-02$ & $4.250313 \mathrm{e}-05$ & $2.625514 \mathrm{e}-09$ & $1.676437 \mathrm{e}-14$ \\
0.40 & $2.631780 \mathrm{e}-01$ & $1.269348 \mathrm{e}-02$ & $5.972126 \mathrm{e}-05$ & $5.163286 \mathrm{e}-09$ & $2.378098 \mathrm{e}-13$ \\
\hline
\end{tabular}

Proof We have

$$
\begin{aligned}
z(t) & \leq \int_{0}^{t} \frac{C}{\sqrt{t-s}} z(s) d s+\int_{0}^{t} \frac{\tilde{C}}{(t-s)^{\alpha}} K s^{m} d s \\
& \leq \int_{0}^{t} \frac{C}{\sqrt{t-s}} \tilde{K} t^{m+\alpha} d s+\int_{0}^{t} \frac{\tilde{C}}{(t-s)^{\alpha}} K s^{m} d s
\end{aligned}
$$

for $0 \leq t \leq T$ and $\frac{1}{2} \leq \alpha<1$. We claim that

$$
\begin{aligned}
& 2 \tilde{K} C t^{m+\alpha} T^{1 / 2}+\tilde{C} K t^{m+\alpha} \int_{0}^{1} \frac{\theta^{m}}{(1-\theta)^{\alpha}} d \theta \leq \tilde{K} t^{m+\alpha}, \\
& \tilde{K} t^{m+\alpha}\left(1-2 C T^{1 / 2}\right) \geq \tilde{C} K t^{m+\alpha} \int_{0}^{1} \frac{\theta^{m}}{(1-\theta)^{\alpha}} d \theta .
\end{aligned}
$$

It suffices to take

$$
\tilde{K}:=K \frac{\tilde{C}}{1-2 C T^{1 / 2}} \int_{0}^{1} \frac{\theta^{m}}{(1-\theta)^{\alpha}} d \theta
$$

The authors declare that they have no competing interests. 


\section{Acknowledgements}

This work was supported by the National Science Center (Poland) decision no. DEC-2011/02/A/ST8/00280.

Received: 31 January 2014 Accepted: 9 April 2014 Published: \#PUBLICATION_DATE

\section{References}

1. Darken, LS: Diffusion, mobility and their interrelation through free energy in binary metallic systems. Trans. AIME 174, 184-201 (1948)

2. Kirkendall, EO: Diffusion of Zinc in Alpha Brass. Trans. AIME 147, 104-110 (1942)

3. Holly, K, Danielewski, M: Interdiffusion in solids, free boundary problem for $r$-component one dimensional mixture showing constant concentration. Phys. Rev. B 50, 13336-13346 (1994)

4. van Dal, MJH, Gusak, AM, Cserhati, C, Kodentsov, AA, van Loo, FJJ: Microstructural stability of the Kirkendall plane in solid-state diffusion. Phys. Rev. Lett. 86, 3352-3355 (2001)

5. Danielewski, M, Wierzba, B: Thermodynamically consistent bi-velocity mass transport phenomenology. Acta Mater. $58,6717-6727(2010)$

6. Bellman, R: Methods of Nonlinear Analysis. vol. II. Academic Press, New York (1973)

7. Bellman, R, Kalaba, R: Quasilinearization and Nonlinear Boundary Value Problems. Am. Elsevier, New York (1965)

8. Mlak, W, Schechter, E: On the Chaplyghin method for partial differential equations of the first order. Ann. Pol. Math. 22, 1-18 (1969)

9. Koleva, MN, Vulkov, LG: Two-grid quasilinearization approach to ODEs with applications to model problems in physics and mechanics. Comput. Phys. Commun. 181(3), 663-670 (2010)

10. Chaplygin, SA: Collected Papers on Mechanics and Mathematics. Moscow (1954)

11. Kantorovich, LV, Akilov, GP: Functional Analysis in Normed Spaces. Pergamon, Oxford (1964)

12. Friedman, A: Partial Differential Equations of Parabolic Type. Prentice Hall International, Englewood Cliffs (1964)

13. Kiguradze, I: On solvability conditions for nonlinear operator equations. Math. Comput. Model. 48, 1914-1924 (2008)

\#DIGITAL_OBJECT_IDENTIFIER

Cite this article as: Leszczyński and Wrzosek: Iterative methods for ternary diffusions. Boundary Value Problems \#CITATION

\section{Submit your manuscript to a SpringerOpen ${ }^{\circ}$ journal and benefit from:}

- Convenient online submission

- Rigorous peer review

Immediate publication on acceptance

Open access: articles freely available online

- High visibility within the field

- Retaining the copyright to your article 